Revista Calidad en la Educación Superior

Programa de Autoevaluación Académica

Universidad Estatal a Distancia

ISSN 1659-4703

Costa Rica

revistacalidad@uned.ac.cr

Autoevaluación y Gestión curricular en el contexto de la Universidad Estatal a Distancia

Self-assessment and curriculum management in the context of the State

University Distance Learning

\author{
Dr. Javier Cox Alvarado \\ Doctor en Educación \\ Universidad Estatal a Distancia \\ San José, Costa Rica \\ Dra. Aurora Trujillo Cotera \\ Doctora en Educación y en Odontología \\ Universidad de la Habana y Universidad de la Salle \\ Cuba y San José Costa Rica
}

Volumen 1, Número 1

Mayo 2010

pp. 1 - 12

Recibido: abril, 2010

Aprobado: mayo, 2010 


\title{
Autoevaluación y Gestión curricular en el contexto de la Universidad Estatal a Distancia
}

\section{Resumen}

El proceso de autoevaluación de un programa o carrera tiene un punto medular el cual es el Plan de Estudios. La autoevaluación de una carrera o programa debe partir de preguntas fundamentales, tales como:

¿Es pertinente la carrera o programa con los requerimientos de la sociedad? ¿Las asignaturas de la carrera o programa proveen a los graduados los conocimientos suficientes en cuanto a pertinencia y actualidad del mercado laboral? ¿Los materiales de los cursos están actualizados? ¿Los cursos se encuentran adecuadamente diseñados para la modalidad a distancia?

Una vez subsanadas las anteriores inquietudes internas a la carrera, se podrá iniciar con el abordaje de las dimensiones externas a ella, tomando en cuenta que una carrera nació con la finalidad de formar un profesional, la cual necesita constantes cambios para amoldarse a una sociedad en constante cambio.

Palabras clave: autoevaluación, plan de estudios, currículo.

\begin{abstract}
The process of self evaluation of a degree program has a very important aspect: the curriculum. The self evaluation of a degree program must begin with fundamental questions such as:

Is it pertinent the degree program with society's requirements? Do the courses provide the graduates enough knowledge regarding pertinence and the present situation? Are the courses of the degree program updated? Are the courses adequately designed for distance education?

Once that the former questions are answered, the process of assessing the external dimensions of a degree program can be initiated, taking in account that that program was born with the aim of forming a professional, and that such degree program needs constant changing to adjust to a society in constant change.
\end{abstract}

Key words: self evaluation, degree program, curriculum.

\section{a. Introducción}

El presente documento tiene como finalidad facilitar el proceso de la autoevaluación de la dimensión currículo de un plan de estudios de una carrera o programa en la Universidad Estatal a Distancia. En su elaboración se han tomado en cuenta 
documentos oficiales de esta Universidad como el Modelo Pedagógico, el Reglamento de Gestión Académica, la Guía para la autoevaluación de la calidad de Programa de Autoevaluación Académica y el documento "Principios teóricos y lineamientos prácticos del diseño curricular en la UNED de Costa Rica".

\section{b. El Plan de Estudios y el currículo}

Según el glosario de la Red Iberoamericana para la acreditación de la Educación Superior (RIACES), el plan de estudios es la Organización de un programa según asignaturas, materias, créditos, cursos y grupos docentes. Se relaciona directamente con el concepto de currículo que también se denomina estructura curricular, es decir el conjunto de asignaturas o materias así como los requisitos académicos con los que se organiza una carrera.

El currículum tiene tres dimensiones fundamentales:

-El diseño curricular. (Planeamiento)

-El desarrollo

curricular. (Ejecución)

-La evaluación curricular.

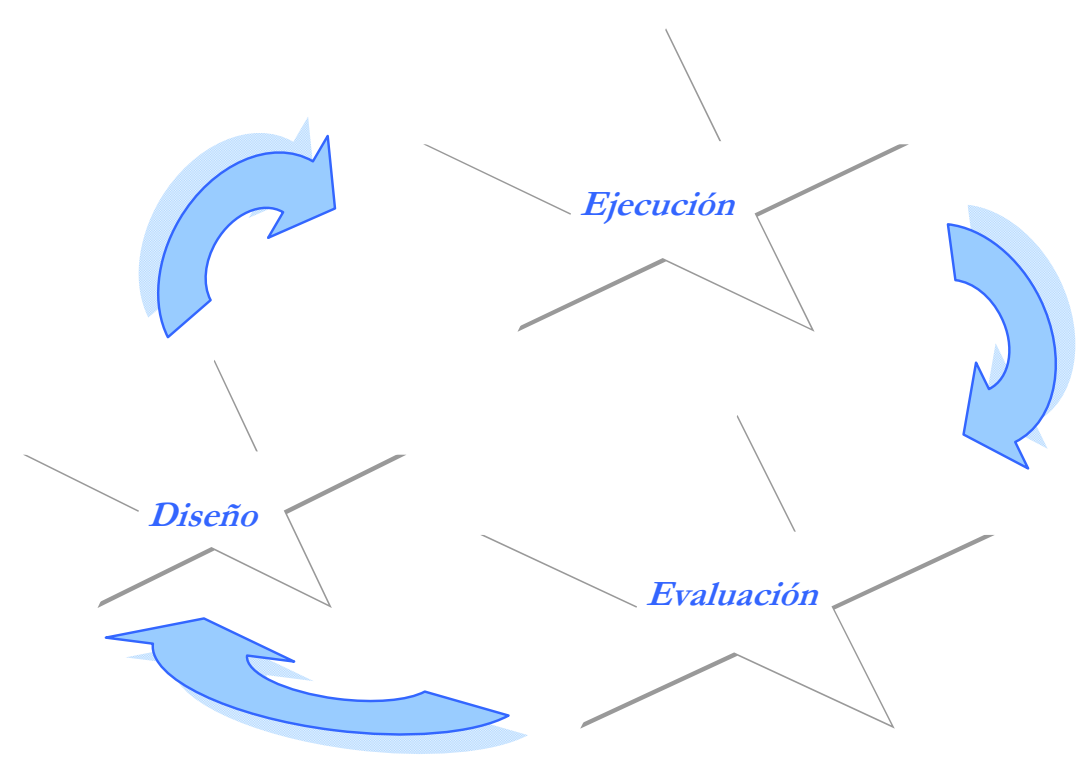

Los diseños curriculares actualmente son elaborados con la participación de diversos actores sociales, especialmente graduados, empleadores, académicos y estudiantes, estos últimos piezas claves para el desarrollo y la concreción del currículo, en tanto se convierten en los agentes activos del desarrollo curricular, y son capaces de realizar 
una práctica educativa orientada hacia la reflexión, la participación, el trabajo cooperativo y la innovación. Ofreciendo nuevas posibilidades en la generación de conocimientos, donde la interdisciplinariedad, el autoaprendizaje y la toma de decisiones, entre otros se tornan en aspectos relevantes de esa formación.

Se visualiza como una totalidad, en la cual se articulan dos aspectos: la realidad social dinámica y la propuesta educativa. Su construcción no puede verse como algo acabado sino como un trabajo flexible y dinámico de revisión y ajuste acorde con un proceso cotidiano de la práctica educativa.

\section{c. El Planeamiento curricular}

En el Reglamento de Gestión Académica de la UNED, es donde se define el planeamiento curricular de una carrera o programa, es una etapa de diseño: del plan de estudio y el diseño de los cursos; los cuales son más conocidos dentro del ambiente universitario de la UNED como Macroprogración y Microprogramación, respectivamente. Implica realizar procesos de selección, organización y evaluación de contenido curricular, de acuerdo al paradigma escogido.

Es necesario destacar que para la elaboración del plan de estudios de una carrera deben tomarse en cuenta una serie de cuestionamientos, los cuales inician desde la pertinencia de impartir una carrera, la necesidad del mercado, los conocimientos que se deben brindar para formar adecuadamente al profesional, la inserción laboral; aspectos que deben cuestionarse permanentemente en un sociedad que se encuentra en cambio permanente.

\section{d. Elementos que debe comprender un Plan de Estudios}

Según el artículo 9 del Reglamento de Gestión Académica, el diseño de un plan de estudios debe comprender al menos los siguientes elementos que se articulan entre si: 
- Justificación del apertura de la carrera o programa, que determina la respuesta pertinente y oportuna a las características del contexto social y a las necesidades en su ámbito académico y profesional,

- Fundamentación teórica-metodológica de la carrera o programa, especificando su marco epistemológico,

- Caracterización del campo de acción del futuro graduado,

- Metas de formación y objetivos de la carrera,

- Perfil de entrada del estudiante y de salida como graduado,

- Línea y estructura curricular, que relaciona y organiza los elementos fundamentales de la etapa de diseño,

- Propósito y descripción de los cursos,

- Recomendaciones para la administración de la carrera o programa.

\section{e. La Justificación de apertura de una carrera o programa}

Es la respuesta de la universidad a las características del contexto social y a las necesidades de la carrera en un campo especifico. En este apartado debe hacerse referencia a la necesidad de formar profesionales en un área específica, se deben tomar en cuenta para ello el resultado de estudios de demanda ocupacional, de mercado, de empleabilidad; lo cual reflejaría la actualidad y pertinencia de una carrera o programa.

Otros aspectos importantes por tomar en cuenta son: la opinión de expertos, los criterios de los colegios profesionales, asociaciones profesionales y organizaciones del área. Asimismo deben analizarse ofertas educativas similares y prever mecanismos para actualizar constantemente la carrera o programa. 


\section{f. La Fundamentación teórico y metodológica de la carrera o programa}

Determinar el marco epistemológico de una carrera significa, reflexionar tanto a cerca del objeto de estudio, construido a partir de la interacción entre los actores del proceso curricular, como de la finalidad del conocimiento. Incluye la relación de la disciplina propia de la carrera con otras disciplinas, las áreas disciplinarias y los ejes curriculares que estructuran un campo de estudio particular y el abordaje metodológico de conocimiento. Para este apartado es necesario demostrar que la carrera o Programa es congruente con los principios y propósitos de la educación a distancia. Para ello se debe revisar la Misión, Visión y Modelo Pedagógico de la universidad sean los que presenta la carrera o programa. Asimismo deben revisarse otros aspectos como la congruencia entre la oferta y las políticas universitarias.

Una vez considerado el marco teórico, se deben buscar los contenidos propios de las asignaturas y adecuarlas a este modelo. Se deben tener claridad en el objeto de estudio, o sea, el conocimiento que se pretende desarrollar, su finalidad, las áreas disciplinarias y la evaluación del programa o carrera.

\section{g. Caracterización del campo de acción del futuro graduado}

Atiende aspectos fundamentales, tales como la relación universidad-sociedad, es decir, la relación entre las necesidades sociales y la formación universitaria para el desempeño profesional. Es necesario que se reflexione sobre la formación que se da al futuro graduado esta debe ser acorde a las disposiciones legales, de tal manera que no se incurra en el ejercicio de otro profesional. Por ejemplo la formación de un contador debe apuntar a un profesional con un perfil diferente a la de un graduado en Banca y Finanzas o Derecho. Debe ser congruente con el perfil y los objetivos. 


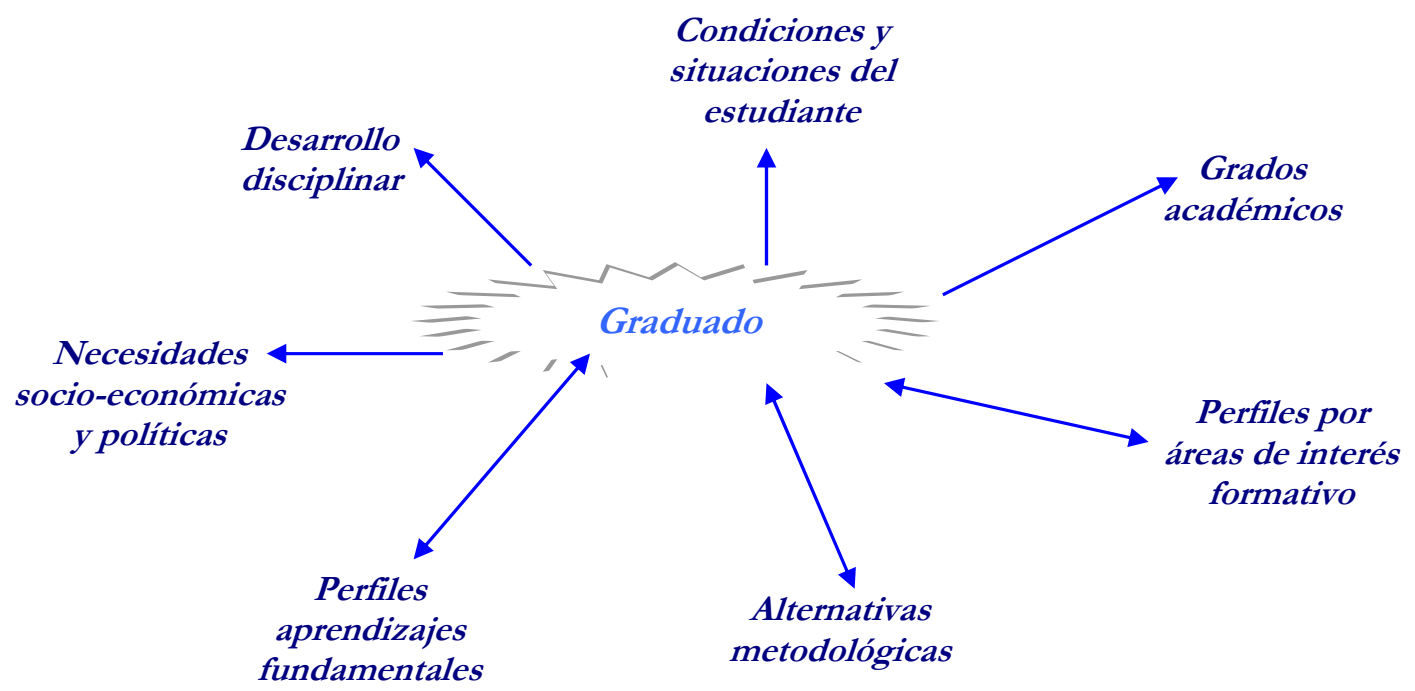

\section{h. Metas de formación y objetivos de la carrera}

Las metas de formación son criterios cuantitativos que se proponen en un plan de estudio en términos de ingreso, promoción y graduación de sus estudiantes, por un período determinado. Están directamente relacionados con los objetivos de la carrera y los recursos disponibles; es necesario que una carrera o programa por ejemplo el número de promociones que tendrá el programa o carrera, si el programa tendrá un periodo definido en su oferta, los cupos disponibles, su definición, cuándo y cómo se realizará.

Se refiere a los "productos" de la carrera o programa, por ejemplo: número de graduados, tiempo de graduación, impacto, producción académica.

Los objetivos de la carrera son enunciados que determinan la identidad de la carrera. Ellos surgen del análisis y la convergencia entre: necesidades socio-económicas y políticas, grados y títulos, desarrollo disciplinar y condiciones y situaciones del estudiante. 


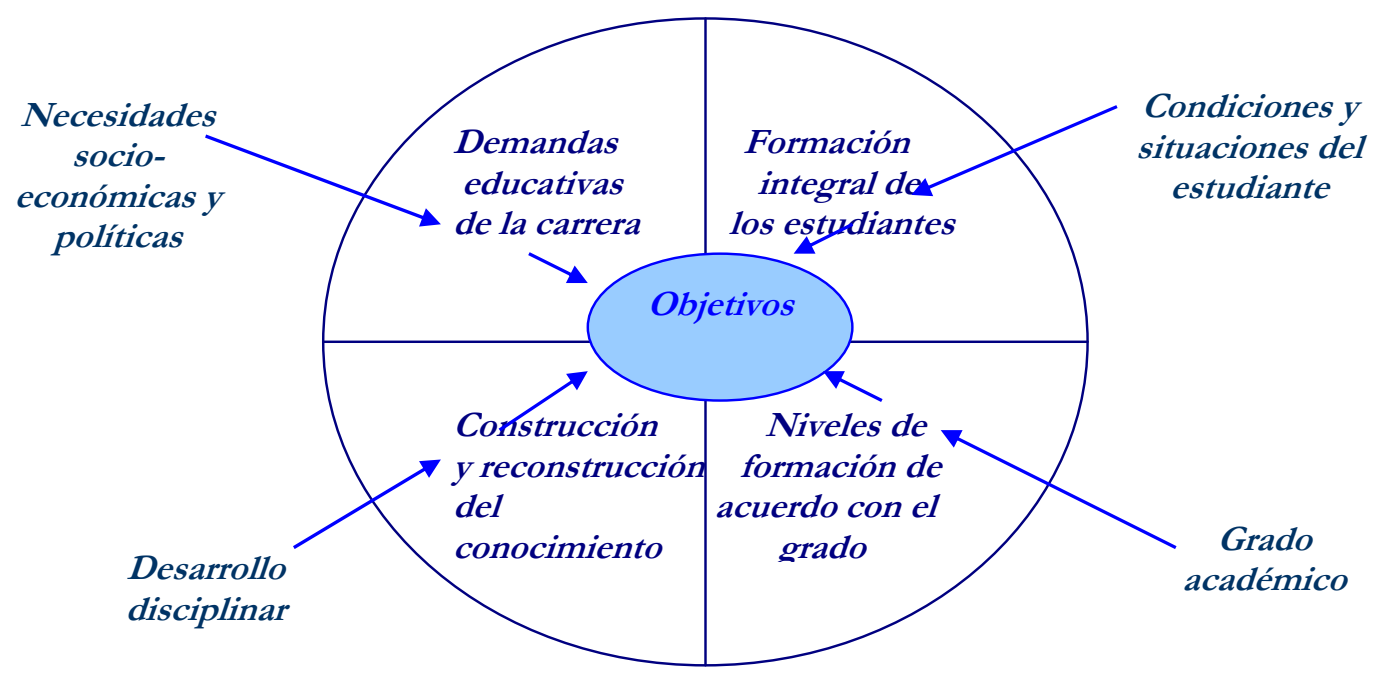

\section{i. Perfil de entrada del estudiante y de salida del graduado}

En relación con el perfil de entrada del estudiante, son todos los requerimientos que establece una carrera o programa para su ingreso. Se expresan las características idóneas que debe tener un estudiante para ser un profesional exitoso en una determinada carrera o programa.

En lo referente al perfil de salida del graduado es la descripción minuciosa de las características que el plan de estudios ha transmitido al estudiante en su proceso de formación. Debe tener total congruencia con el campo de acción del futuro graduado

\section{j. Línea y estructura curricular}

La línea curricular permite visualizar el contexto de una carrera como un continuo, una línea con dirección y sentido que persigue una determinada meta en la formación de los graduados. Conduce necesariamente a cuestionarse sobre la oferta existente y sobre la necesidad de una nueva oferta académica oportuna y pertinente, en tanto responda a las características del entorno y a las necesidades de formación del contexto social. 
La estructura curricular organiza y relaciona los elementos fundamentales de la etapa de diseño curricular a partir de sus funciones. Origina una dinámica interna que integra los conocimientos propios de la carrera.

En este apartado es importante reflexionar sobre la correspondencia entre la estructura curricular y el perfil profesional, los contenidos de los cursos son los suficientes para cumplir con el perfil profesional, existe un adecuado número de cursos y número de créditos. ¿Ha sido revisada la línea y estructura curricular por expertos tanto el área del currículo como por expertos en el área de formación del programa o carrera?

Debe tenerse claridad en las habilidades que el estudiante irá adquiriendo y establecer secuencias al organizar los contenidos de aprendizaje y las actividades diseñadas para la enseñanza y el aprendizaje.

\section{k. Propósito y descripción de los cursos}

Al reflexionar sobre el propósito del curso, va a permitir conocer el propósito de cada uno de los cursos en la formación del estudiante dentro del plan de estudios.

Además en este apartado debe aparecer la descripción de todos los cursos que componen el plan de estudios. Debe explicar el "qué" y "para qué" del mismo.

Es importante recordar que cada descripción de curso debe contar con la siguiente estructura:

- Código

- Naturaleza

- Nivel

- Créditos

- Horas totales

- Requisitos 
- Objetivo general:

- Objetivos para el estudiante

- Temática

- Bibliografía básica

\section{Recomendaciones para la administración de la carrera o Programa}

El diseño curricular especifica medidas administrativas en términos de organización, ejecución y control.

Dada la naturaleza de la UNED es necesario reflexionar sobre aspectos básicos en un sistema a distancia como lo son:

- La Mediación pedagógica

- El cumplimiento de las orientaciones para el curso (Cronograma de actividades)

- Velar por un adecuado diseño y producción de la Unidad Didáctica Modular (materiales impresos y electrónicos), así como la actualización de ésta.

- El conocimiento de necesidades de poblaciones

- Un adecuado sistema de evaluación formativo y sumativo.

- Diseño de cursos o actividades en entornos virtuales de aprendizaje (plataformas de aprendizaje en línea, videoconferencia, teleconferencia).

\section{m. Los tres elementos fundamentales para el análisis, elaboración y reelaboración de un Plan de Estudio}

Para analizar un Plan de estudio es importante tener claridad en tres elementos que se interrelacionarán en todo momento, ellos son la Congruencia Interna, pertinencia y 
actualidad. La Congruencia Interna se puede observar por ejemplo al concordar el objetivo de la carrera con el perfil de graduado y la existencia de cursos que formen al profesional con las características requeridas. La pertinencia la encontramos al analizar constantemente las exigencias del mercado y sus requerimientos. La actualidad va muy ligada a la pertinencia, pero se enfoca mayormente en estar a la vanguardia en conocimientos, materiales u otros, que mantengan a una carrera o programa al día. Una práctica común es evaluar el perfil profesional de una carrera o programa cada cinco años.

\section{n. Conclusiones}

El análisis del Plan de Estudio es el punto medular en la autoevaluación de una carrera o programa. Es necesario tener en cuenta que una carrera o programa, nace para responder a una necesidad identificada, la cual es la formación de profesionales en un área determinada. La función social de la universidad debería ser el análisis de las necesidades de formación y de inserción de los profesionales en el mercado laboral. Por lo tanto es necesario que los planes de estudio, los perfiles y las asignaturas no puedan ser elementos estáticos, deben ser flexibles y estar en un constante cambio. Este proceso tiene como referente la concepción de un diseño curricular que fortalece sistemáticamente la generación de conocimientos, y orienta la práctica de manera articulada y coherente, en pos de una formación integral. El currículo es un elemento vivo, tan vivo como el elemento en el cual se centra en la UNED: EL ESTUDIANTE. 
Bibliografía

Álvarez Zayas, Carlos. (1995) Fundamentos teóricos de la dirección del proceso de formación del profesional de perfil amplio. Universidad Central de las Villas. La Habana, Cuba.

Amino, S. et al. (2002). Towards a Shared Vision for Higher Education. CrossCultural Insights \& Proyects. University of Kassel: Germany.

CEMPA. (2004). Principios teóricos y lineamientos prácticos del diseño curricular en la UNED de Costa Rica". UNED.

Programa de Autoevaluación Académica. (2008) Autoevaluación de la calidad de carreras de educación a distancia. Inédito

Taba Hilda. (1962). Curriculum development: theory and practice. New York, NY: Harcourt, Brace and World.

Universidad Estatal a Distancia. (2005) Reglamento de la gestión académica de la UNED. EUNED.

Sitios Web consultados:

http://www.riaces.net/glosarioo.html

http://www.uned.ac.cr/paan/

http://www.uned.ac.cr/Reglamentos/reglamentos/docs/ESTATUTO\%200RGANIC

O.doc 